\title{
Sexyloop: Self-Reproduction, Evolution and Sex in Cellular Automata
}

\author{
Nicolas Oros Chrystopher L. Nehaniv \\ School of Computer Science, University of Hertfordshire, College Lane, Hatfield Herts AL10 9AB, United Kingdom \\ \{N.Oros,C.L.Nehaniv\}@herts.ac.uk
}

\begin{abstract}
We created a simple evolutionary system, sexyloop, on a deterministic ten-state five-neighbour cellular automaton (CA) where self-reproducing loops have the capability of sex. With this ability, the loops are capable of transferring genetic material into other loops. This work was based on Sayama's evoloop which was transformed by adding a new state and new rules. The evoloop model showed an emergent evolutionary process only due to an adaptation of the loops to interaction in the environment; and after a certain time, all the individuals capable of self-reproduction belonged to the smallest species 4 which reproduced the fastest.

We created two different models of self-reproducing loops with sex, Sexyloop M1 and M2, in order to study the possibility of sex in self-reproducing automata and to assess the impact of sex on the evolutionary process via comparing between the evoloop and the sexyloop variants. In the sexyloop $M 1$ and M2, the diversity of the whole population was different from that found in the evoloop and the evolutionary process was quite different too. The sexyloops also created smaller and bigger species than the evoloops, and exhibited greater diversity and faster evolution than their non-sexual counterparts. The most interesting model was the sexyloop M2 whose evolutionary dynamics had a very different longterm behaviour than the evoloop and sexyloop M1. The most surprising and intriguing phenomenon in the sexyloop M2 was that the evolutionary process was selecting quickly a bigger species than in the evoloop and sexyloop M1: the species 5. In fact, the individuals from this species needed more time to reproduce than those from the species 4 . So it appears that in the sexyloop M2, the fittest individual was not one that could reproduce the fastest but surely one that reproduced fast and which was more adapted to propagate in an environment where sex with individuals of the same and other types could occur. These results give the first examples in cellular automata of evolution in a population of self-replicators where sex plays an important role.
\end{abstract}

\section{Introduction}

This paper reports the first implemented work on sex in selfreproducing cellular automata (CA). CAs are regular arrangements of cells where each cell has a state. The cells' behaviours are defined by deterministic rules which modify the state of a cell depending only on the states of the cells in its neighbourhood (cells around it) and/or depending on its previous state. There are mainly two types of neighbourhood for two-dimensional CAs, the von Neumann and the Moore neighbourhood. CAs were created by John von Neumann and Stanislaw Ulam by the end of the 1940s as a tool to address the possibility of self-replication in machines [11]. Von Neumann tried to understand what are the sufficient features needed by an automaton to reproduce itself or even produce something more complex. He used a universal Turing machine embedded in a cellular array using a 5-cell neighbourhood (the cell itself plus the four adjacent cells) and 29 possible states per cell. This machine creates a configuration of states in an array by reading from a tape the information corresponding to the structure. The created structure is in fact a copy of this universal constructor and its input tape. "[T]here are two levels of automaton in this construction: 1) the cellular automaton itself (the array); and 2) the universal constructing automaton which is embedded in the cellular automaton as a configuration of states. Thus a configuration can be automaton itself" [2]. Doing this, von Neumann succeeded in realizing the first non-trivial (embedded) self-replicating configuration in a cellular automaton [11]. An important property of von Neumann's construction was to consider that the instructions present in the tape should be used in two different ways: (1) uninterpreted and (2) interpreted [2]. Indeed, it is remarkable that von Neumann's solution used genetic, inherited information in two roles: (1) blindly copied and (2) executed, before the structure of the heritable genetic material in life on earth was uncovered by Watson and Crick [6].

Von Neumann's model is very complex because of the size of the self-reproducing configuration as well as due to the large number of states per each cell. So around 1968, E. F. Codd created a simplified model of a self-reproducing automaton using just 8 states per cell based on von Neumann's work [1]. One of Codd's most important ideas was to create what he called a "periodic emitter" consisting of a data path forming a loop where the data can travel inside like a signal. Due to this structure, a finite data stream can be made to always turn inside the loop at regular intervals. With this idea, the static tapes containing the data could be replaced by loops situated in the CA that allow the data to be stored permanently. In 1984, Langton modified the structure of Codd's periodical emitter to create a simple and efficient self-replicating loop (SR loop) using only 8 states locally and a five-neighbour CA space. The signal circulating in the emitter was interpreted to create an arm allowing the creation of the new loop's body and uninterpreted when it was copied into the new loop. The the data signal served as heritable genetic information, a genome, directing the self-reproduction of the loop. This structure could replicate itself in just 151 updates and rotate by 90 degrees counter-clockwise to create copies of itself in four places all around [2]. We can make an analogy [9] to biological systems where the genotype (genetic information 
or genome) of the SR loop is the signal and the phenotype (structure or shape) includes the loop structure (Q-shape tube structure) and its behaviour.

In 1998, Sayama modified Langton's SR loop by adding a ninth dissolving state [8]. This state could make the loops disappear (die) in their environment allowing a continuous self-reproduction and turn-over of generations in a finite space. He named this model the structurally dissolvable selfreproducing loop (SDSR loop). Then, Sayama created the evoloop based on the SDSR loop using also nine states [9]. The very interesting feature of this model was the fact that the population of loops could actually evolve in a finite space even though no explicit evolutionary mechanism was incorporated. The emergent evolutionary process was due to direct interactions of the phenotypes that would sometimes modify the genotypes of loops. This kind of process is different from random mutations and could be suggested as an important process that occurred in ancient times and modified primitive living forms [9]. In the evoloop model, after a certain time, the smallest loops are naturally selected to be the main species in the population due to the fact that they replicate faster than the others. Sayama's work "gives an affirmative answer to the question of whether it is possible to construct an evolutionary process- [...] as a process in which self-replicators vary and fitter individuals are naturally selected to proliferate in the colony-by utilizing and tuning up a simple deterministic cellular automata" [9].

Self-reproduction process of an evoloop proceeds in stages:

- create a long arm (part of which will become the first side of the new offspring)

- $\quad$ turn the tip (end) of the arm by 90 degrees

- $\quad$ build the second side of the offspring loop

- $\quad$ turn the tip (end) of the arm by 90 degrees

- $\quad$ build the third side of the offspring loop

- $\quad$ turn the tip (end) of the arm by 90 degrees

- $\quad$ build the last side of the offspring loop

- join the last side of the offspring loop to the parent's arm

- dissolve the umbilical cord (arm) between parent loop and offspring

- create a new sprout of the arm

- create a long arm, etc...

\section{Sexyloop: A Self-Reproducing Loop Capable of Sex}

\subsection{Concepts}

In order to compare loops in the evolutionary process, Sayama defined loop "species". He labelled the name of the species depending on the number of '7' states ("genes") situated in the genome signal. For example, a loop from species 5 has five ' 7 ' genes, species 6 has six ' 7 ' genes and so on. Several different variants of same sized species can exist (generally depending on the location of a pair of ' 4 ' genes in the genome - controlling turning of the arm tip relative to the '7' genes).
In the evoloop, the loops could evolve to larger and smaller species. Some loops could also lose their self-replicating ability entirely or even produce smaller loops; after a certain time, the population was mainly composed of the smallest loops able to reproduce, species 4 . This model showed an emergent evolutionary process only due to an adaptation of the loops to their environment. Interactions were just collisions occurring in the environment, and there were no functional interactions between individuals that could modify the diversity of the population.

We made the choice to modify the evoloop so a loop could be able to transfer its genetic material into another one. With this ability, the diversity of the evolving population as a whole was expected to be different from that found in the evoloop.

Let us now refer to characterizations of sex:

- "There are two aspects of human sexual reproduction that are universally found in all sexual creatures: recombination and outcrossing. Recombination refers to the physical breakage and rejoining of DNA molecules. Outcrossing refers to the fact that the DNA molecules involved in recombination come from two different individuals from the previous generation: our mother and father." [5]

- "Sex in the biological sense, as we pointed out earlier, means simply the union of genetical material from more than one source to produce a new individual" [4] (cf. also [3])

- "Sex is in biology, by definition, nothing more than the transfer or exchange of genetic material." [6]

Since the self-reproducing loops, from Langton's to Sayama's, have genetic material in the form of a travelling signal, according to the characterizations of sex given above, we can consider that the evoloop model we modified could be said to support a capability for sex. So we refer to this new model as Sexyloop.

\subsection{The Sex Connection}

We wanted to allow the transfer of genetic material from a loop into another one using a simple mechanism with a minimum number of new states, using a mechanism similar to bacterial conjugation [4]. So we needed to identify a special configuration when one loop hits another one. In the evoloop, all undefined rules create a dissolving state ' 8 '. When the tip of a loop's arm hits another loop on its sides or the corners, a dissolving state appears eventually deleting the "attacked" loop and the attacker's arm. We mean by "attacker" the loop that will transfer its genetic material into another loop (the "attacked" loop). The use of the term "attacker" in this paper is due to the fact that in our scenario, the partner transmitting heritable information sexually is at an evolutionary advantage to the recipient as the latter generally loses some part of its genome in such interactions. We do not believe this to be a 
general property of sex, but a limitation of the current haploid self-replicating loop models which have only very limited space to house genetic material.

For the Sexyloop, we decided that the attacker's arm should bond with the attacked loop, creating a bonder state ' 3 ' on its sheath (Figures $1 \& 2$ ). This junction was only made if the attacker's arm hit another loop on its side, not on the corners. So when a loop hit another one at a corner, its behaviour was the same as in the evoloop. We wanted to keep the collisions at a corner like in the evoloop because this is one of the most common collisions leading to dissolution happening in the environment allowing a continuous self-reproduction in a finite space.

Then, we had to find a way to transfer the signal from the attacker loop into the attacked one. In fact, we managed to do it by adding just one new state ' 9 ' with different functions and the corresponding rules (Table 1). With this new state, we had to create different kind of rules.

The first set of rules we created was to allow genetic transfer, the second one was to delete an arm when it has corners (which could not occur in evoloop but arose frequently in sexyloop), and the last set of rules was for increased robustness. We needed the last set because with sex, many special configurations could happen at the same time such as two loops or more having sex together, a take-over of the arm with a loop that is having sex, etc. (Figure 3). We created these sets of rules for two different mechanisms of sex.

\subsection{First Sex Mechanism}

We named the sexyloop using the first mechanism sexyloop M1. An important fact is that in this mechanism, the transfer is made ONLY when the beginning of the attacked loop's signal arrives at the junction. If the signal start has already moved past where the junction is created (as in Figure 1), the attacking loop will wait for the signal to come back around in order to transfer its genes. So until then each state in the copy of the genetic material composing the signal coming into the attacker's arm is deleted when it touches the bonder ' 3 '.

Once the beginning of the attacked loop's signal (corresponding to a state ' 7 ' or ' 4 ' sometimes) arrives at the ' $T$ ' junction, a state ' 9 ' is created in the middle to block (delete) the attacked loop's genetic signal as it arrives (Figure 4). Thereupon, the blocker ' 9 ' moves from one cell in the direction from where the attacked loop's signal comes, to allow the transfer of genetic signal sent by the attacker. At the same time, the bonder ' 3 ' disappears and the incoming signal moves forward (Figure 5).

The transfer begins and a detection sheath ' 9 ' is created to detect when the incoming signal ends (Figure 6). During transfer, the signal blocker ' 9 ' will always be situated between a core cell ' 1 ' and the transferred signal. When the genetic material coming in from the attacker has been transferred, then only core cells ' 1 ' - which come at the end of the genome in the ancestral loops - are present at the junction with the attacker's arm (Figure 7).

Then, an umbilical cord dissolver ' 6 ' is created in the attacker's arm beside the detection sheath. A blocker dissolver ' 9 ' is also created to delete the signal blocker (Figure 8). Finally, the umbilical cord dissolver moves back into the attacker arm to retract it and a sheath ' 2 ' is created in its previous location. At the same time, the signal blocker, the detection sheath and the blocker dissolver disappear (Figure 9).

The genome of the attacked loop has thus been modified so the loop has a great chance to belong to another variant species (except if the new genome is exactly the same as the old one). Due to the connection and synchronization mechanisms required for sex, the signal transferred by the attacker can have various sizes. It depends on where the signal is situated in the arm when it bonds with the other loop and depends on the length of the genetic material that the signal is made of. If the genetic signal present in the attacked loop is contiguous and has an equal or smaller number of genes than the transferred part of the attacker's signal, it will be completely replaced by the new material. Otherwise, only a part of the signal of the attacked loop will be deleted. In this case, a little space (core cells ' 1 ') will be created between the old signal and the new section since it takes a few time steps to delete the connection made for sex.

\subsection{Second Sex Mechanism}

This model of sexyloop using the second mechanism was named sexyloop M2. The difference between the first and second mechanism is the synchronization process used for the genetic transfer. In the first mechanism, the transfer was started only when the beginning of the recipient loop's signal arrives at the junction. We decided that the second mechanism should be more flexible. While the sexyloop M1 can only start the transfer of their genes from the time the attacked loop's signal arrives at the junction, sexyloop M2 can begin the transfer at any time until the end of the signal arrives at the junction.

This mechanism of sex increased the probability of the attacker loops transferring more genetic material and leads to a more diverse recombination of heritable material. This also made the sexual transfer faster because the attacker did not have to wait for the beginning of the genome of the attacked loop to come around if the junction had already been established.

When the sex connection is created, any genes (' 7 ' or ' 4 ') arriving at the ' $\mathrm{T}$ ' junction are transformed into a signal blocker ' 9 ' (Figure 10). Then, the mechanism works like in the sexyloop M1. 


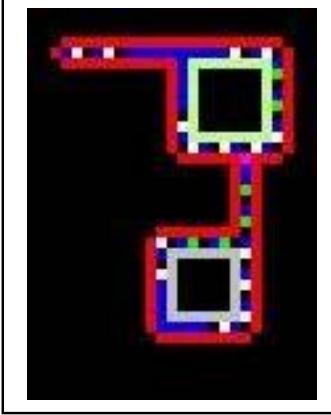

Figure 1. Sex junction on the side of the "attacked" loop

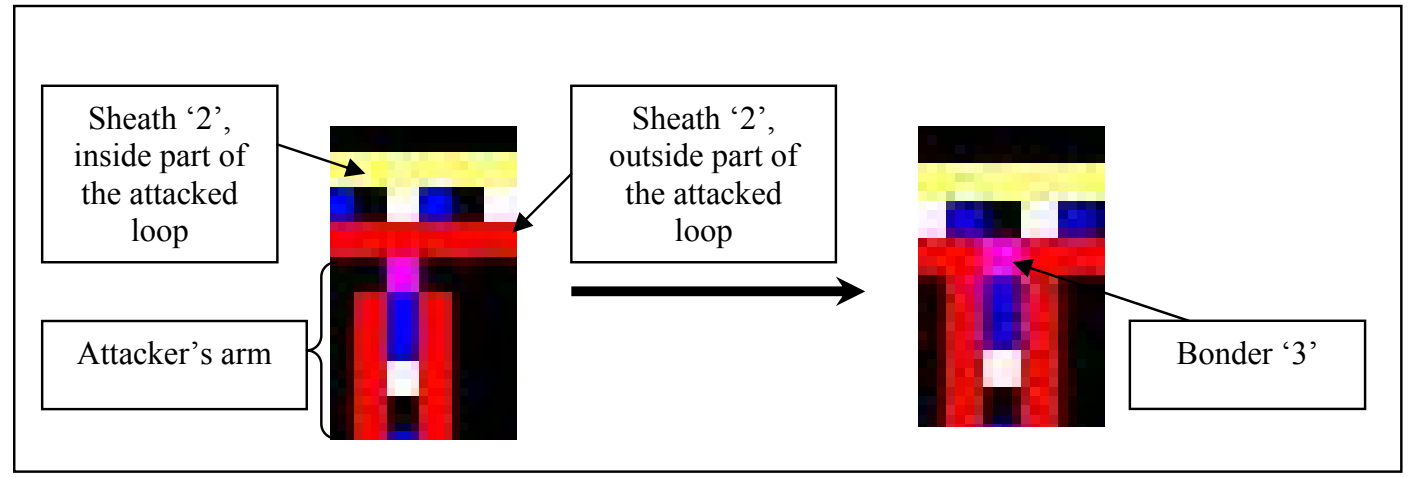

Figure 2. Junction between the arm and the loop

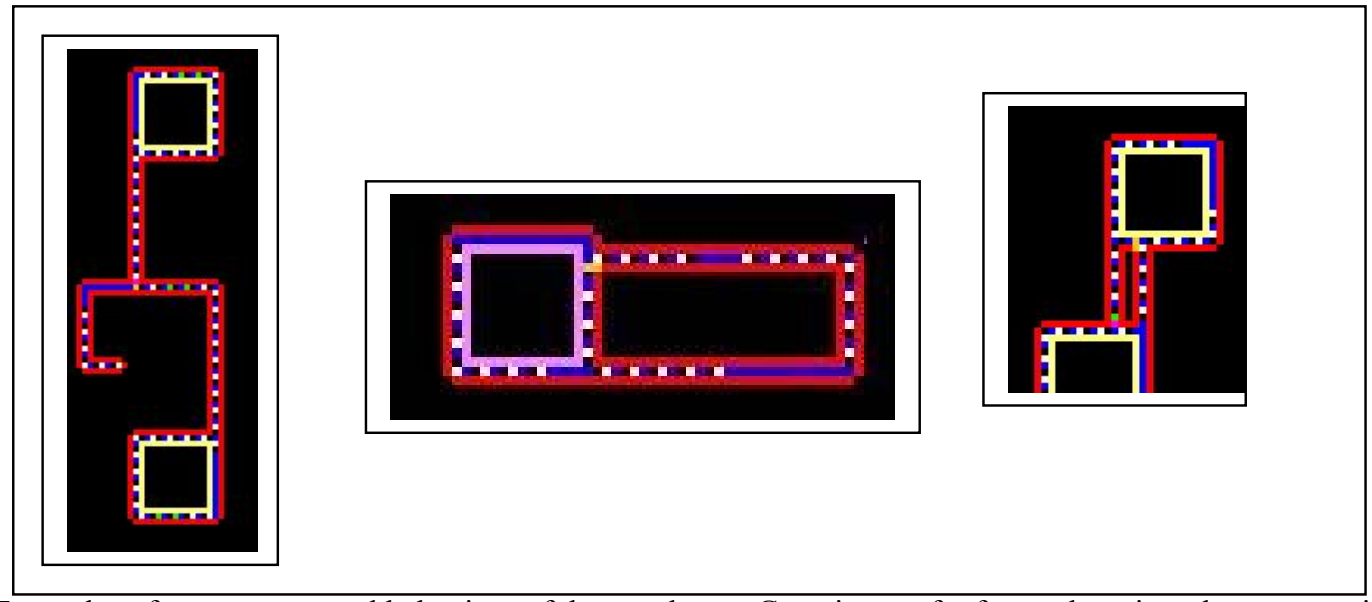

Figure 3. Examples of emergent sexual behaviour of the sexyloops: Genetic transfer from a loop into the constructing arm of another loop (left). Genetic transfer from a loop into itself (middle). Two loops having sex together (right).

\begin{tabular}{|lll|}
\hline $\begin{array}{l}\text { State } \\
9\end{array}$ & $\begin{array}{l}\text { Name } \\
\text { Signal blocker } \\
\text { Detection sheath }\end{array}$ & $\begin{array}{l}\text { Functions } \\
\text { Stop a signal from being conducted in the loop } \\
\text { Detect the end of the transfer (necessary to create an arm } \\
\text { dissolver in the attacker's arm) } \\
\text { Delete the signal blocker }\end{array}$ \\
\hline
\end{tabular}

Table 1. Names and functions of the additional states in the CA of the Sexyloop 


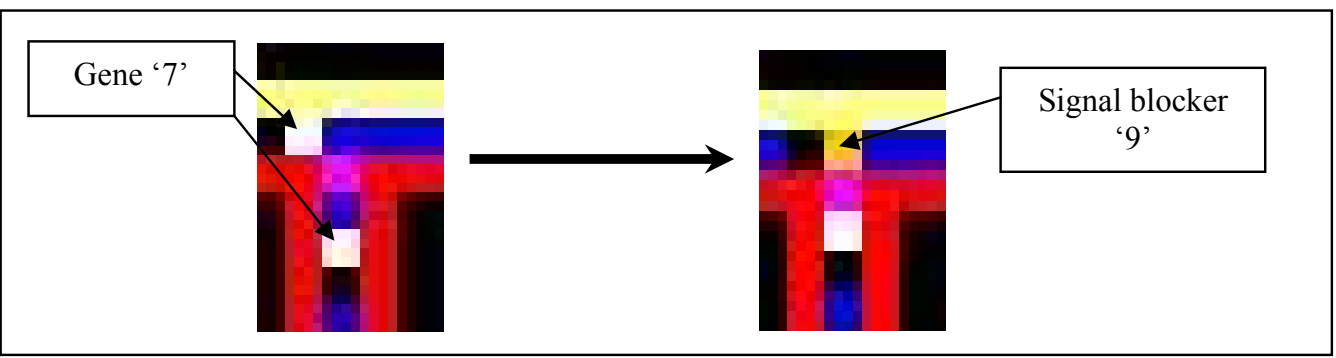

Figure 4. Creation of the signal blocker
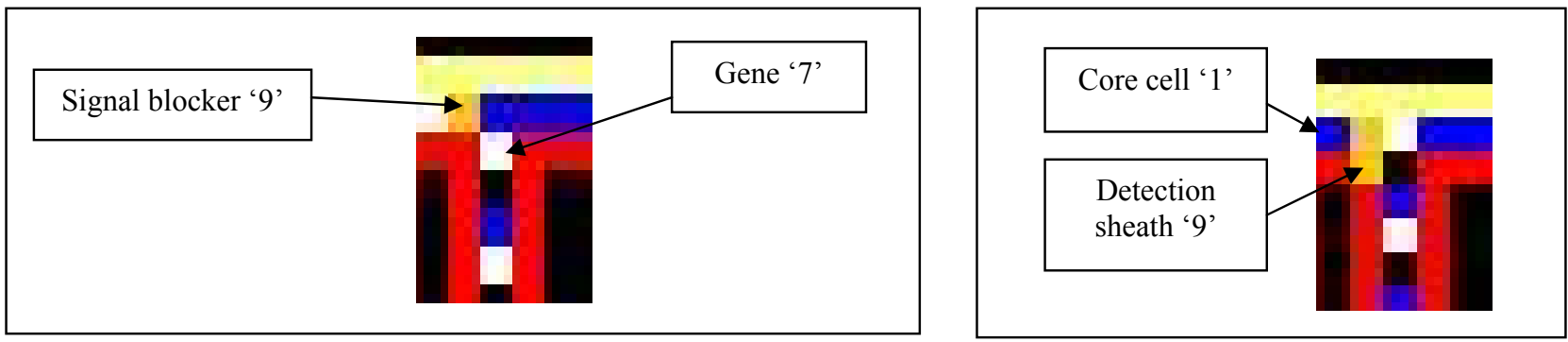

Figure 5. Beginning of genetic transfer

Figure 6. Creation of the detection sheath

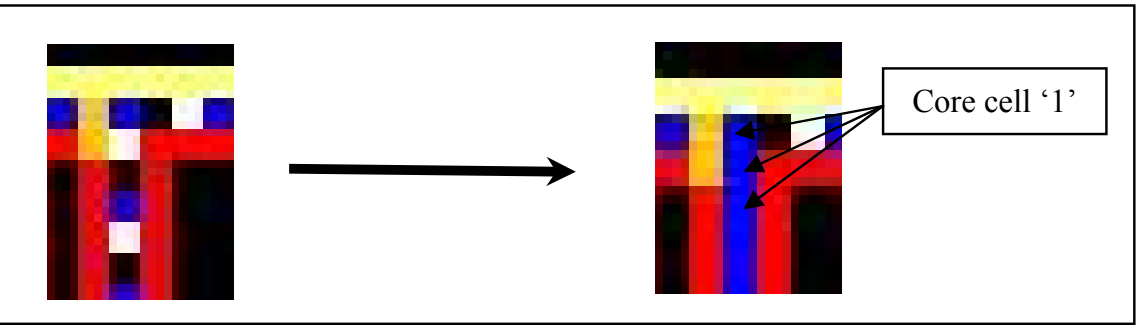

Figure 7. End of genetic transfer

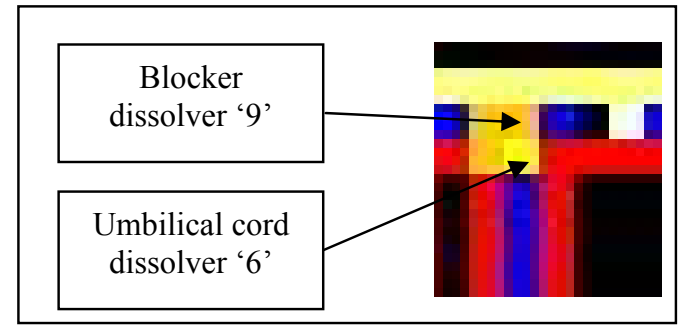

Figure 8. End of sex connection

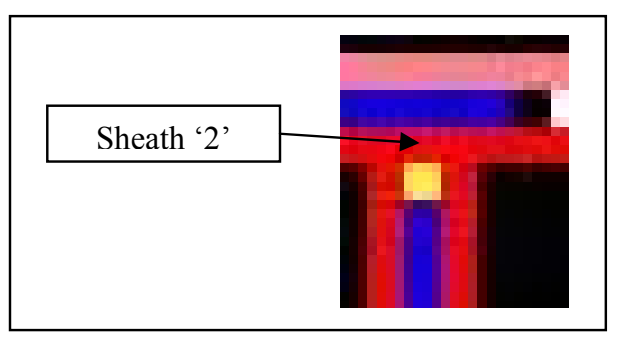

Figure 9. Dissolving the attacker's arm

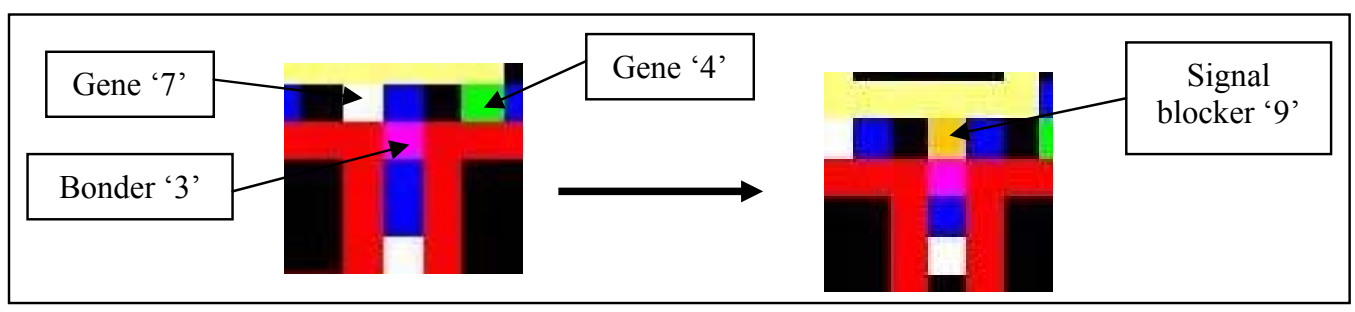

Figure 10. Sex in the sexyloop M2 


\section{Experiments}

We have chosen to use an ancestor loop of species 13.2. Following Sayama's notation [9], '13.2' means the subspecies of species 13 (with 13 ' 7 ' genes) with a pair of ' 7 ' states situated before a pair of ' 4 ' genes. We remark that for this species, the two ' 4 ' genes are situated near the beginning of the signal, so the attacked loop had more chance to lose its self-replicating ability during sex especially for the sexyloop M1 (a pair of ' 4 ' genes is necessary for self-replication). But the attacker loop could still give the attacked loop its own genes ' 4 ' depending on where they were situated when the connection was made.

We decided to run ten different simulations with the evoloop, the sexyloop M1, and the sexyloop M2 making a total of 30 simulations on toroidal grids, using ten space sizes: 100, 200, 300, 400, 500, 600, 700, 800, 900 and 1000 (e.g., space 100: $100 \times 100$ sites) with all the runs traced until 50,000 updates. With these simulations, we were able to analyse the different properties of the three different models in order to compare them. Results using a space of $400 \times 400$ sites and a space of $1000 \times 1000$ sites are shown here as examples. Figures 11 and 12 show the distributions of average number of individuals belonging to each species over the entire run, for sizes 400 and 1000 , respectively. Tables 2 and 3 record the average number occurrences per timestep of asexual reproduction and of sexual reproduction via sex into loop arms or sex into (other parts of) loops for these respective space sizes.

\subsection{Experiments using a space of $400 \times 400$ sites}

Species occurring for evoloop at space size 400 ranged from 3 to 14,1 to 16 for sexyloop M1, and 1 to 17 for sexyloop M2. Figure 12 shows the average number of individuals per species per timestep.

In the evoloop, the dominant species of the system that evolve from the species 13 was the species 4 . In this case, the species 4 was clearly the dominant species where the species 5 population was decreasing to become almost extinguished. In this experiment, the species 4 was composed of two main subspecies having different genomes.

In the sexyloop M1, the dominant species of the system that evolved from the species 13 was also the species 4 . In this case, the species 4 was clearly the dominant species and the species 5 population was never really very prevalent.

Surprisingly, in the sexyloop M2, the dominant species of the system that evolve from the species 13 was the species 5. We noticed that the species 4 population was quite prevalent but never took over from the species 5 population. In this experiment, the species 5 was composed of two main subspecies having different genomes.
When we compared the fittest individuals from species 4 and 5 found in the evoloop and in the sexyloop M1 and M2, we could see that their genomes were nearly all different. The only species which had the same genome were the species 5 from the evoloop, the sexyloop M1, and the secondary subspecies 5 of the sexyloop M2. The main subspecies 5 in the sexyloop M2, which was the fittest, was only found with this model.

\subsection{Experiments using a space of $1000 \times 1000$ sites}

Species occurring for evoloop at space size 1000 ranged from 2 to 16,1 to 18 and 21 for sexyloop M1, and 1 to 20 for sexyloop M2. Figure 13 shows the average number of individuals per species per timestep.

In the evoloop, the dominant species of the system that evolve from the species 13 was again the species 4 . In this case, the species 5 was the dominant species until the species 4 population became quite prevalent at around 40,000 time steps. Then the population of species 5 decreased quickly. In this experiment, the species 4 and the species 5 were composed of three main subspecies each having different genomes.

In the sexyloop M1, the dominant species of the system that evolved from the species 13 was also the species 4 . In this case, species 4 was clearly the dominant species and the species 5 population appeared to decrease very slowly from 33,000 time steps (earlier than for evoloop). In this experiment, the species 4 and 5 were each composed of three main subspecies having different genomes.

In the sexyloop M2, the dominant species of the system that evolved from the species 13 was the species 5 . In this case, the species 4 and the species 6 were prevalent in the population but could never take over the whole population. In this experiment, the species 5 was of higher diversity, composed of five main subspecies having different genomes, and the species 4 was composed of three main subspecies.

By comparing the fittest individuals from species 4 and 5 found in the evoloop and in the sexyloop M1 and M2, we could see that some genomes are different and some are similar. All the subspecies 4 from the evoloop (except the third) were present in the sexyloop M1 and M2. The main subspecies 5 in the evoloop was present in the sexyloop M1 but not in the M2. The second subspecies was found in all the models but the third one was only present in the sexyloop M2. The sexyloop M1 and M2 had one similar subspecies not present in the evoloop. Evolutionary transitions toward smaller species occurred more rapidly with the sexual loops M1 and M2, and diversity was higher than with the non-sexual evoloops. 
Figure 11. Distribution of species for the evoloop, sexyloop M1 and M2 averaged over 50,000 time steps (space size:400 x 400; bars show standard deviation).

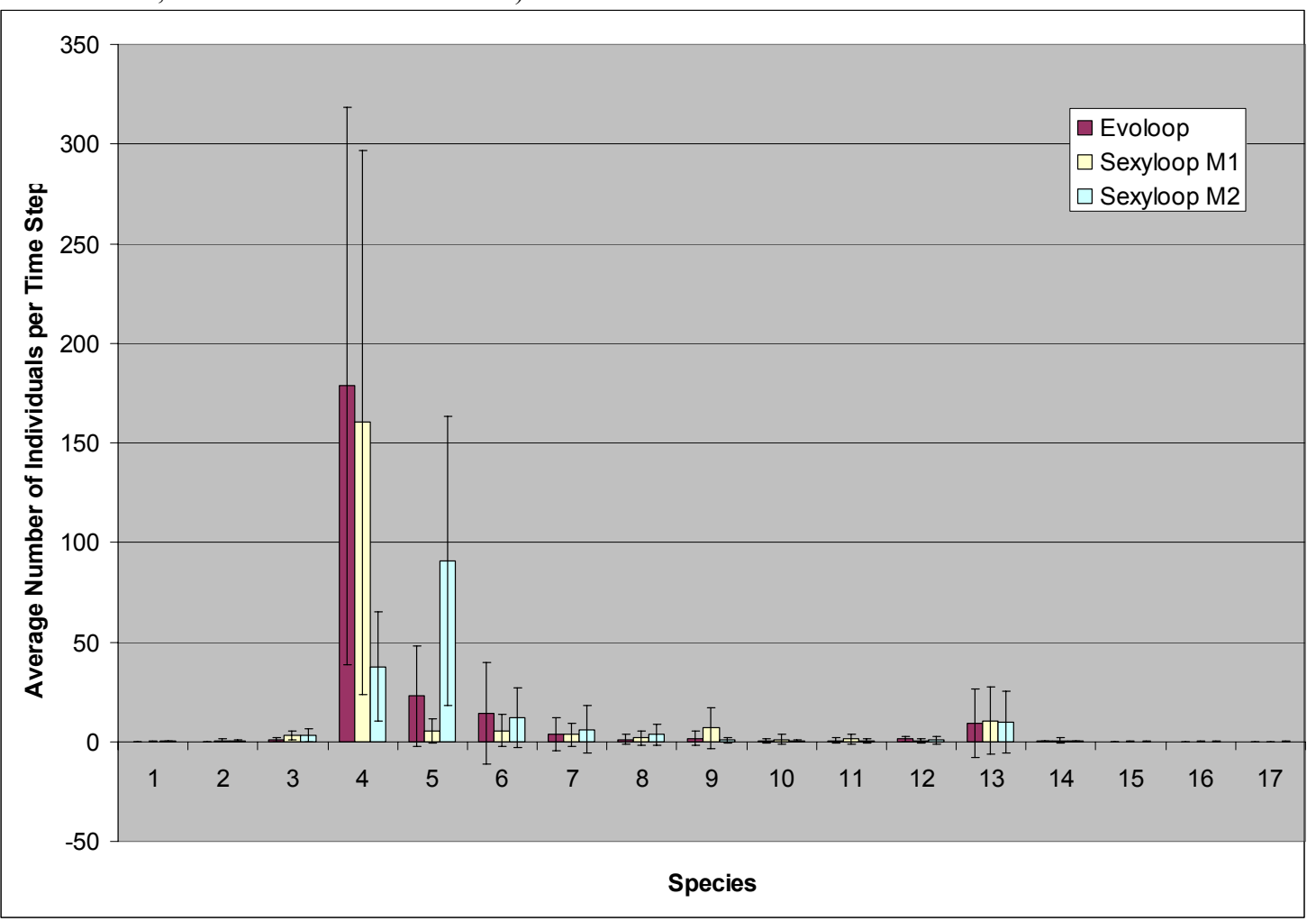

Figure 12. Distribution of species for the evoloop, sexyloop M1 and M2 averaged over 50,000 time steps (space size:1000x1000; bars show standard deviation.)

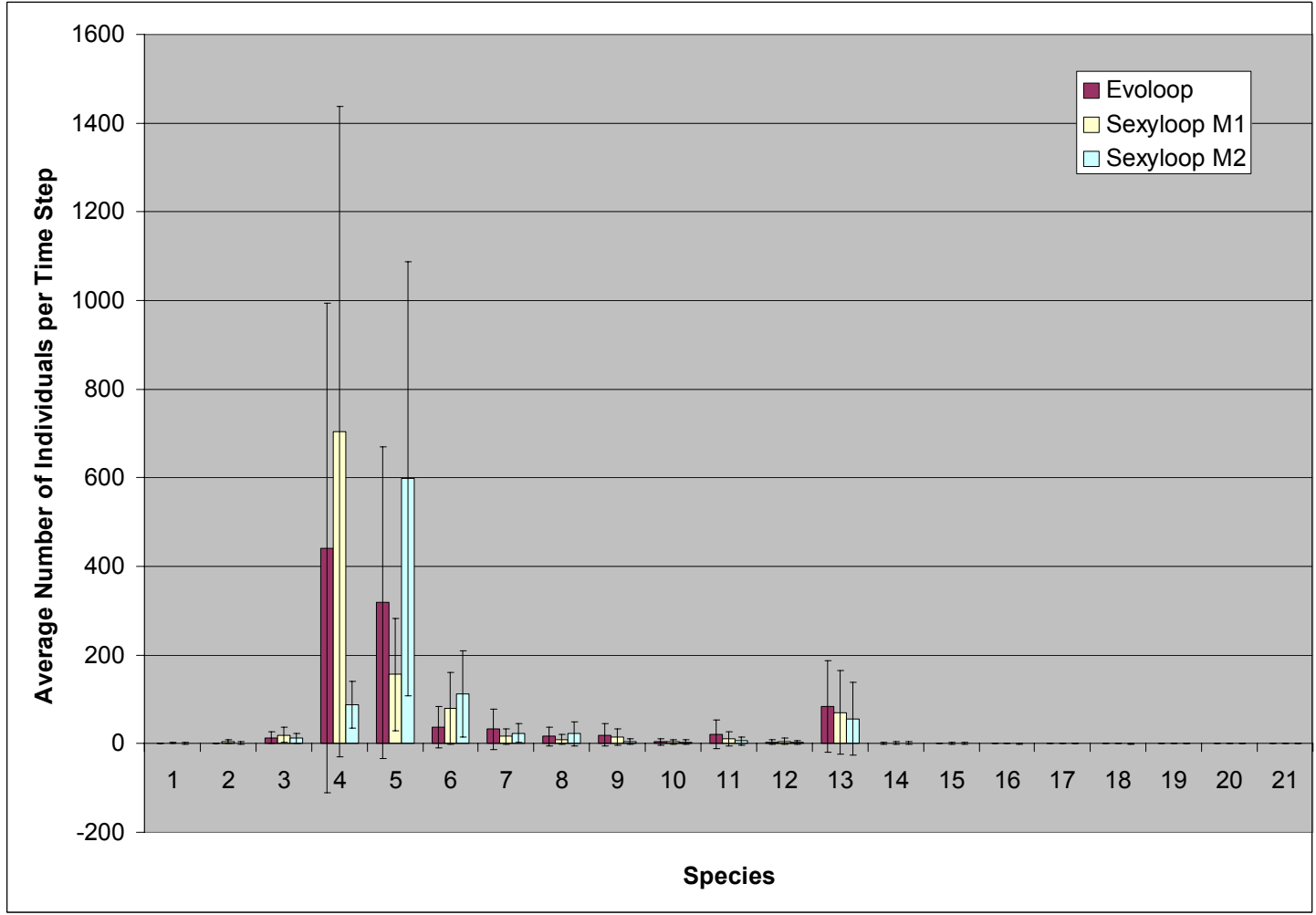


Table 2. Mean instances of sexual and asexual reproduction over time with standard deviation in the population for 50,000 time steps (Space size 400).

\begin{tabular}{|c|c|c|c|c|c|c|}
\hline & \multicolumn{2}{|c|}{ Evoloop } & \multicolumn{2}{c|}{ Sexyloop M1 } & \multicolumn{2}{c|}{ Sexyloop M2 } \\
\cline { 2 - 7 } & mean & s.d. & mean & s.d. & mean & s.d. \\
\hline $\begin{array}{c}\text { Asexual } \\
\text { reproduction }\end{array}$ & 115.31 & 70.78 & 95.08 & 71.99 & 65.96 & 39.88 \\
\hline Sex into arm & 0 & 0 & 0.397 & 0.651 & 0.285 & 0.540 \\
\hline Sex into loop & 0 & 0 & 0.818 & 1.056 & 0.634 & 0.903 \\
\hline
\end{tabular}

Table 3. Mean instances of sexual and asexual reproduction over time with standard deviation in the population for 50,000 time steps (Space size 1000).

\begin{tabular}{|c|c|c|c|c|c|c|}
\hline & \multicolumn{2}{|c|}{ Evoloop } & \multicolumn{2}{c|}{ Sexyloop M1 } & \multicolumn{2}{c|}{ Sexyloop M2 } \\
\cline { 2 - 7 } & Mean & s.d. & mean & s.d. & mean & s.d. \\
\hline $\begin{array}{c}\text { Asexual } \\
\text { reproduction }\end{array}$ & 425.26 & 385.36 & 484.1 & 407.2 & 349.6 & 220.1 \\
\hline Sex into arm & 0 & 0 & 2.365 & 1.975 & 1.524 & 1.473 \\
\hline Sex into loop & 0 & 0 & 5.718 & 3.795 & 4.000 & 3.306 \\
\hline
\end{tabular}

\section{Discussion}

With these different experiments, we could see that the sexyloop M1 did not differ a lot from the evoloop in that it was always the individuals from the species 4 that were the fittest after 50000 time steps. We supposed that this first sex mechanism did not modify the genomes of the different loops enough to radically change the evolutionary process.

In contrast, the sexyloop M2 seemed to clearly change the evolutionary process by modifying the genomes of the individuals, and in every case, the loops from the species 5 were naturally selected. Sexual populations of sexyloop M1 and M2 apparently evolve more quickly than for the non-sexual population (represented by evoloop), and incidences of sex continued for the sexual loops throughout the evolutionary process.

We had to notice that in the sexyloop M2, the fittest individuals had different genomes depending on the size of the environment compared to the fittest individuals of the evoloop where the subspecies genomes tended to be the same across environments. To summarize, there were more different subspecies 5 in the sexyloop M2 than subspecies 4 in the evoloop and some of these subspecies 5 seemed to be more adapted to certain environments than other. The fittest individuals from the species 5 living in a small environment could have a different genome than an individual from the same species living in a larger environment. We ran some tests using the evoloop simulator to compare how fast one loop could selfreproduce. We selected the two fittest individuals from the species 4 and four fittest ones from the species 5 (found in the sexyloop M2). As suspected, the loops from the species 4 reproduced always faster than the individuals from the species 5. So there should be other reasons why the species 5 was dominant. It seems that in the sexyloop M2, the fittest individual was not necessarily one who could reproduce the fastest (as in the evoloop [9]) but rather one most adapted to survive (with other species) in its environment and perhaps also most adapted for sex transfer. In fact, just by comparing the genomes of the fittest individuals, the reasons why the species 5 is dominant in the sexyloop M2 remain elusive.

In most of the experiments using the sexyloop M2, the species 4 remained quite prevelant even if the species 5 was dominant. We suspect that the loops mainly from species 4 and 5 might have found a way to co-evolve in the environment by modifying their genomes. Even in extended runs (lasting 100,000 timesteps) for all sizes, species 5 always persisted and was (except in the $400 \times 400$ and $700 \times 700$ cases) dominant. The evolutionary process occurring in the sexyloop M2 appeared to be faster than in the evoloop and skipped some steps in the process having less different dominant species. In fact, the species 5 dominated more quickly the other species in the environment.

We have to notice that sex, in the sexyloop, is costly in time since it depends on the shape and the positions of the loops at a particular time, and a loop could be killed before transferring copies of its genes into another one (especially with the sexyloop M1). On the other hand, the alternative in evoloop was usually dissolution due to collision. Sex had fitness costs too when it created small species (not present in the evoloop) that could not reproduce.

Biologists believe that a major role of sex is to repair genetic material [5]. Unfortunately, sex has a very low probability to just effect repair in the sexyloop because although a loop might repair another loop that has lost its '4' genes with sex by transferring the missing genes, it also can transfer genes that the damaged loop did not have before. In fact, we suspect that future self-reproducing loops with the ability to repair genes will need to have a template matching mechanism with two copies of the genetic material (diploidy).

There is no male and female in the sexyloop - all the loops in the sexyloop were genderless. Reproduction generally occurred asexually but sexyloops also had the capability to transfer genetic material into another loop by having sex. Unlike usual bacterial conjugation, sex in the current CA models usually involves the destruction and replacement of part of the genetic material of the recipient by sexually transferred material. It is conceivable that creation of a new loop could sometimes occur in evoloop or either of the sexyloop models by mutual "take-over" of the arms of two interacting loops and that the new loop could have parts of the genomes of both "parents" - however, as far as the authors know, such an event has never been observed and, if possible, seems likely to be very rare. 
Sex in the sexyloop is just a particular configuration triggered based on environmental configuration, dependent on CA rules rather than something carried in the genomes of loops. The connection used for sex was created by using a gene ' 7 ' which was also used to grow the arm of a loop straight. The loops did not have any specific "sex gene" used to create this connection. In living creatures, such a sex genes do exist so it would be natural to modify the sexyloop by adding a gene in the genome of the loop which would be used only for inducing sexual behaviour, and to study its persistence or extinction in evolving populations.

\section{Conclusion}

The main goal of this project was to implement sex into Sayama's evoloop. The evoloop model showed an emergent evolutionary process only due to an adaptation of the loops to the physical environment. Interactions were just collisions occurring in the environment and there were no functional interactions between individuals that could modify the diversity of the population [9]. This is why we made the choice to modify the evoloop so a loop was able to transfer heritable information into another one, a simple form of sex.

We created the two different models (sexyloop M1 and M2) of self-reproducing loop capable of sex. These models are the first to the implement sex in selfreproducing loops on cellular automata. Vitányi [10] created theoretical models of sexually reproducing CA but he never implemented them and his models were based on von Neumann's work using tapes which is different from Sayama's loop. Sex can occur in Ray's self-reproducing programs in Tierra [7], bearing some similarity to our mechanisms but unlike in sexyloop, it does not occur between two "living" individuals and is not necessarily local in space. We noticed that in the sexyloop, the diversity of the whole population was different than that found in the evoloop and the evolutionary process was quite different too. The sexyloop M2, which we think was the most interesting model, had a very different behaviour than the evoloop and sexyloop M1. The most surprising phenomenon was that the evolutionary process of the sexyloop M2 was selecting a bigger, slower replicating species than in the evoloop and sexyloop M1: the species 5 . The reasons why such phenomenon appears remain to be elucidated.

The examples here provide the first implemented instances, as far as we know, of sex occurring in selfreproducing configurations in cellular automata. It would be natural to explore alternative realizations of sex, especially those with less destructive means of transfer or recombination of heritable genetic material.
Future works should carry out more tests using the sexyloop M2 with different ancestors and using much larger environments to verify whether the behaviours we described are always observed. An efficient tool to measure the diversity of the population by comparing genomes between species and subspecies should be developed in order to have a better comparison of the different models. We suggest to modify the sexyloop by adding a separate sex gene into the genome which will be used only to create the sex connection. With such model, we could study whether or not this sex gene is passed from parent to offspring in various configurations and investigate whether and in what circumstances sex survives in the course of evolutionary dynamics.

\section{References}

1. Codd, E. F. (1968). Cellular Automata. ACM Monograph Series. Academic Press, New York.

2. Langton, C. G. (1984). Self-reproduction in cellular automata. Physica D, 10:135-144.

3. Margulis, L and Sagan, D. (1986). Origins of Sex: Three Billion Years of Genetic Recombination, Yale University Press.

4. Margulis, L and Sagan, D. (1987). Microcosmos: Four Billion Years of Evolution from Our Microbial Ancestors, Harper Collins.

5. Michod R. E. (1994). Eros and Evolution: A Natural Philosophy of Sex. Helix Books. Addison-Wesley Publishing Company.

6. Nehaniv C. L. (2005). Self-Replication, Evolvability and Asynchronicity in Stochastic Worlds. In: O. B. Lupanov et al. (Eds.), Stochastic Algorithms, Foundations and Applications (SAGA), LNCS 3777, pp. 126-169, Springer-Verlag Berlin Heidelberg.

7. Ray, T. S. (1992). An Approach to the Synthesis of Life, Artificial Life II, Santa Fe Institute Studies in the Sciences of Complexity, Proceedings Volume X, C. G. Langton (Ed.), Addison-Wesley, Redwood City, California, pages 371-408.

8. Sayama, H. (1998). Introduction of structural dissolution into Langton's self-reproducing loop. In: C. Adami, R. K. Belew, H. Kitano, \& C. E. Taylor (Eds.), Artificial Life VI: Proceedings of the Sixth International Conference on Artificial Life, pp. 114122. Cambridge, MA: MIT Press.

9. Sayama, H. (1999). A new structurally dissolvable selfreproducing loop evolving in a simple cellular automata space. Artificial Life, 5(4): 343-365

On-line material at:

http://necsi.org/postdocs/sayama/sdsr/\#software

10. Vitányi, P. M. B. (1973). Sexually Reproducing Cellular Automata. Mathematical Biosciences 18: 2354.

11. Von Neumann, J. (1966). Theory of Self-Reproducing Automata. Edited and completed by A. W. Burks. Urbana: University of Illinois Press. 